\title{
THE BOWEL AS A FOCUS OF SEPSIS IN CHRONIC RHEUMATIC DISEASE.
}

\author{
By JAMES W. WILTSIE, A.B., M.D. \\ (Consultant in Physical Therapy at the Binghamton City Hospital, Binghamton, N.Y.)
}

Before considering the bowel as a focus of sepsis in chronic rheumatic disease let us first contemplate chronic rheumatism as a whole, reviewing briefly as many of the predisposing and etiologic factors as we are able, and appraising them as accurately as possible, that we may reach a fair estimate of their relative importance, and in so doing arrive at a fairly accurate idea of the position held by septic foci in the bowel. This having been done we will be better able to indicate logical methods of therapy and ultimately to check the correctness of our reasoning through results of such therapy intelligently applied.

First, just how many disease entities does the term chronic rheumatic disease embrace, and is sepsis in the bowel etiologically significant in all? If we accept the classification of the American Rheumatism Association such specific infections as gonorrhœal, tubercular, and syphilitic arthritis must be included. Obviously sepsis in the bowel would have no etiologic significance in these, other than secondary or contributory. Again, we would be compelled to consider such conditions as backache, sciatica, gout, myositis, fibrositis, bursitis ; allergic, metabolic, and endocrine arthritis; and still others, singly or in multiple. Sepsis in the bowel could certainly be excluded as a direct etiologic factor in some of these but not in all. It would be necessary to consider it in rheumatoid arthritis and possibly in osteo-arthritis. Thus early in our discussion we become aware not only of vague boundaries and overlapping of disease entities occasioned by specific and non-specific infection or by no infection at all, but also of identical entities, ultimate resultants of obviously differing combinations of etiologic factors. Likewise we are cognizant of the fact that infectious foci of identical nature may cause rheumatism in one individual, myocarditis or arteriosclerosis in another, or no disease at all in still a third.

In order to avoid too much confusion by separate consideration of all disease entities embraced by the term chronic rheumatic disease in which sepsis in the bowel might conceivably be a factor, I will arbitrarily limit my remarks to a discussion of the condition commonly known as rheumatoid or atrophic arthritis. It is true that there are those who deny that sepsis or focal infection is an essential factor in the etiology of this disease just as there are authors who deny that there is such a thing as systemic toxæmia of intestinal origin. Yet this or similar groups might also deny that neuritis, fibrositis, bursitis or any other entity of the chronic rheumatism group could be caused occasionally by colon sepsis. Furthermore, many of those who believe that focal infection is a sine qua non of rheumatoid arthritis are unwilling to concede that the bowel may be incriminated as such a focus. With all these differing conceptions it is hard to find a common basis of agreement from which to launch a discussion.

Questions such as these immediately arise. Given a focus of infection in teeth, tonsils, or colon, what are the factors that determine whether the individual will develop rheumatoid arthritis, neuritis, fibrositis, nephritis, intestinal toxæmia, or no disease at all? What may be said of such conditions in which no focus or infection can be found, or having been found and eradicated these conditions fail to disappear? Do biologic laws exist under whose operation any of the above apparent inconsistencies may readily be explained? To answer the last question first, I believe such biologic laws do exist. Furthermore, I believe that much of 
the loss of confidence in the theory of focal infection to explain, in part at least $\frac{2}{2}$ many of our chronic and degenerative diseases, is due largely to our failure to $\stackrel{D}{\circ}$ understand these laws, especially in their inter-relationship and scope.

As a result of many years of study of focal and systemic infection and much $\stackrel{\Rightarrow}{\vec{F}}$ clinical experience in the treatment of such infection and the toxæmias and disease 0 entities resulting therefrom (Wiltsie, I938), I feel that certain important phenomena and processes essential to its establishment and permanent entrenchment have been $\frac{\bar{m}}{\overrightarrow{ }}$ overlooked or their influence greatly underestimated. Foci of infection recognized $\frac{D}{2}$ and removed before secondary foci have had an opportunity to develop are rarely the cause of chronic disease. Allowed to remain too long they become the source $\vec{o}$ of secondary foci and vicious circles whose ramifications and limitations would be $\vec{\overrightarrow{ }}$ difficult to trace, and whose permanency no longer depends upon the continuing $\vec{\rho}_{\mathscr{\sigma}}$ existence of the primary focus. The primary and all known secondary foci may be removed without much hope of successfully ridding the body of infection, since the offending organisms may be distributed rather diffusely throughout the whole $\vec{\sigma}$ body, in lymphatic drainage systems, liver and biliary drainage system and in the intestinal tract which they reach by way of the biliary system or by direct excretion $N$ from the blood stream.

By this time certain tissues of the body become increasingly sensitive to the 9 end-products of bacterial life and death and develop an altered reactivity to these $\vec{T}$ products and those resulting from their union with antibody, which reactivity is $\frac{\mathbb{D}}{\circ}$ commonly spoken of as bacterial allergy. Such highly sensitized tissues have been called the shock tissue, which becomes the tissue in which the characteristic pathology of the disease entity is found. However, all the tissues of the body shor 0 this increase in sensitiveness to the end-product of antigen-antibody union to sones extent as is indicated by the phenomenon of retardation.

About foci of infection it would appear that antibodies of the host unite with antigenic substances, fixing them and thereby rendering them harmless. However, the end-products of this reaction, which I have spoken of elsewhere as immunotoxins, retard drainage by building a protective barrier about the focus. It $\stackrel{\odot}{\circ}$ becomes difficult for bacteria to get out and difficult for antibodies and leucocytes $\overrightarrow{0}$ to get in. As the tissues become increasingly sensitive to these immunotoxins their 3 reaction to them becomes exaggerated to such a degree that a biological stalemate is established. Wherever antigen and antibody meet, an astringent effect is produced which stops or greatly retards the interchange of substances from one side of $\frac{}{3}$ the barrier to the other. Thus the focus is protected and becomes chronic, and if this biological balance is not disturbed by surgical removal of the focus, drainage 3 . or desensitization of the tissues, it may continue in status quo indefinitely.

Allergy, therefore, because of this attribute-retardation, is perhaps the most $\stackrel{3}{\xi}$ important factor making for chronicity where infection is concerned, and without 9 question the greatest hindrance to drainage of tissues. Furthermore, I believe $\frac{}{0}$ sensitization of the involved tissues (the shock tissues) of affected joints to the end-products of antigen-antibody union is a necessary prerequisite to the pathology $N$ of rheumatoid arthritis.

The pathogenic organism itself is not necessarily present in the shock tissue, or if present may be very difficult to find and identify because of the infrequency of its appearance. So great is the sensitivity of shock tissue that the pathologicalo process may continue active with the presence in the body of unbelievably small numbers of living organisms. Foci harbouring these organisms may be entirely quiescent, or diffuse, exhibiting no gross pus, and very difficult to locate. A ${ }_{\bar{O}}^{0}$ slightly enlarged lymph node, an innocent-looking appendix or gall-bladder, $a \underset{\otimes}{\stackrel{\odot}{~}}$ 
normally functioning and normally appearing colon, singly or in multiple may harbour a sufficiently large number of bacteria to cause chronic neuritis, arthritis, or even arteriosclerosis over a period of years.

The organism usually implicated in chronic foci of infection is a streptococcus, yet staphylococci, colon bacilli and others have been isolated in pure culture. However, focal infection is generally considered from the non-specific point of view since different strains of any given organism appear to be able to create identical pathological lesions. It would appear that such factors as the establishment of vicious circles, allergy, and inadequate drainage, both cellular and gross, were the determining factors governing the specific manifestation in which chronic focal infection expresses itself, rather than the nature of the infecting organism and the resistance of the host. In other words tissue reactivity and factors influencing the natural drainage systems are more important etiologic factors in focal infection than the degree of specific immunity attained. In its relation to arthritis the nature of the infecting organism is important only as it affects the preparation of, or selection of, a vaccine for the purpose of desensitizing the tissues to bacterial allergy. This subject will not be discussed more than to say that in all cases of rheumatoid arthritis in which the colon is implicated a routine vaccine should be used. Desensitization of tissues cannot but aid natural drainage.

Any physician who has treated many cases of arthritis is able to recall one or more cases of short duration which cleared up entirely following the removal of a single focus of infection. This is because secondary foci had not yet become established. Cohen (I937) writes: "If foci are removed within six months of the onset of arthritis the patient usually recovers without further treatment." With the establishment of secondary foci we may assume that the blood stream has been invaded, usually by way of the lymphatic drainage from the primary focus, which means failure of this system to control the infection. With blood stream involvement sufficiently great and of sufficient duration to be held responsible for the development of secondary foci the functional threshold of bacteriolysis of the liver must have been overstepped.

With functional impairment of the local lymphatic system and of the liver, bacteria of focal origin soon make their appearance in the intestinal tract. From the first indication of an original focus of infection to the appearance of the causative organism in the bowel an interval of time varying from several months to several years or more may elapse. At this point, however, vicious circles begin to develop. Yet before the colon is thus definitely implicated arthritis may occasionally be cured in a comparatively short time. However, since the colon, once infected, becomes a common link in several vicious circles the cure of arthritis assumes a more serious aspect. The complete eradication of infection now becomes a matter of years rather than months. Not only must all known foci be removed or drained, but these internal vicious circles must be broken and kept broken until every circle has been completely drained of infection. Since the colon is common to at least three circles it would seem to be the logical point of attack. It should, therefore, be kept as free from the presence of the pathogenic organism as possible, continuously, for many months. Inasmuch as allergic retardation, invariably present in arthritic conditions, is a factor definitely interfering with free drainage, this process is slowed down in speed to a fracton of that common to the ordinary case of intestinal toxæmia. Furthermore, many cases of arthritis of unquestioned intestinal origin may show none of the usual symptoms of intestinal toxæmia in spite of the fact that many predisposing causes and underlying pathologic processes are the same, as well as many of the therapeutic indications. 
Those who believe that focal infection plays little or no part in the etiology' of rheumatoid arthritis, as well as those who are unable to find any indication that bacterial allergy is a factor, would find it very difficult to explain Crowe's (I937) experience that of any single method of treatment vaccines give " far and away the best results." It is true that some cases do not respond to vaccine therapy, but this is also true of other diseases in which the infectious etiology cannot be questioned. Failure to respond to vaccine therapy, therefore, cannot be accepted as evidence that rheumatoid arthritis is not due in part at least to focal infection. Furthermore, failure to find foci of infection or to identify the causative organism cannot be accepted as evidence that such do not exist. Lautman (I938) says: "Lack of improvement following the removal of some obvious focus often indicates continued absorption from some lesser and perhaps unsuspected area. If future study reveals that the process is one of bacterial sensitization, it is quite conceivable that continued absorption of the sensitizing substance from bacteria in locations free from active disease may be responsible for maintaining the joint disturbance."

Zinsser and Grinnell (I925) suggest that streptococcus allergy might have some relationship to certain forms of arthritis in which the association of sterile joint conditions with streptococcus infection had been recognized clinically, Harkavy and Hebald (I932) reported nine cases of asthma in adults who had also developed arthritis. In eight of these the arthritis cleared up and the asthma greatly improved following the removal of the foci of infection. These authors state that evidence is rapidly accumulating to the effect that the allergic state is part of the immunologic mechanism accompanying infection. They conclude that "In asthma induced by a chronic focus of infection, bacterial allergy is an admite tedly accepted mechanism. Co-existent arthritis due to the same focus is in al probability an expression of a similar reaction in allergic persons in whom the lungs are the primary and the joints the secondary shock tissues."

Hurst (I9I9) states: "When no obvious source of infection ... . can be found (to account for arthritis) . . . the possibility of an intestinal origin must be considered. . . . The disease has been cured after the removal of an appendix. Many instances of improvement have been recorded following treatment of constipation and chronic colitis by intestinal lavage and other means." Traut (I934) finds streptococci unusually numerous on the colonic mucosa of arthritics and has isolated them from the blood in about 70 per cent. of patients. He says: "Against the bowel wall the pathogens are favoured by heat and moisture and are protected from the bacteriostatic agencies in the bowel lumen. It is probable that these streptococci are true parasites rather than excreta of the colonic wall." Lieb (I929) says that in streptococcal infections of the intestinal tract it is often impossible to demonstrate streptococci in the fæces and concludes: "Our experience justifies the conclusions that focal infections, primarily outside the alimentary tract, play a statistically minor rôle in the production of chronic disease and that wherever the primary infection may be, secondary infections of the gastro-intestinal tract may eventually develop through hæmatogenous or gravitational sources." Keating believes that while streptococci can be cultured from the stools in conditions other than arthritis, the most frequent area of focal infection in arthritis is the gastrointestinal tract, and he reports the culture of streptococci from the stools of the great majority of his arthritics.

It would not be difficult to duplicate this evidence many fold. Burbank (I935) finds streptococci in the stools of 95 per cent. of his arthritics and in a large percentage of blood cultures from these patients. Albee (I928) implicates the colon 
in 90 per cent. of cases of myofascitis. Snyder, Hartsock, Fishbaugh, Wyatt, Gutman, Cohen, Leir, Miltner and Kulowski, Ellman, Copeman, and many others believe colon stasis and sepsis definitely etiologic in rheumatoid arthritis. Pemberton (I932), Fletcher, and others believe malfunction of the intestine as a whole plays a very much more frequent and important rôle in arthritis than do the various "surgical" infective processes. Abnormalities in function and configuration are particularly stressed, such as achlorhydria, carbohydrate indigestion, lethargy of the gall-bladder, dilatations and re-duplications of the bowel, elongation, visceroptosis, atonia, etc. At the present time it would be impossible to say whether many of these conditions were primary and directly etiological or predisposing, or secondary and the result of some common predisposing or causative agent. Chronic deficiency disease could conceivably account for some.

So far, it would appear that the author considers focal infection and bacterial allergy the sole etiological factors in arthritis worthy of consideration. As a matter of fact he is not in the least unorthodox in his convictions concerning the importance of heredity, morphologic type, climatic conditions, occupation, and temperament as predisposing causes, or of nutritional deficiencies, endocrine, nervous or psychic exhaustion, shock, fatigue, exposure, trauma and unrelated or secondary infection as initiating causes. Many of these are determining factors (the answer, in part, to the first question set forth in paragraph four). A number of them are generally present in any given case of arthritis, yet no single one can be considered essential. Infection and allergy, on the other hand, are definitely sine qua non factors in rheumatoid arthritis.

Early in the study of chronic infection I learned that an appreciable number of patients continued to show symptoms of toxæmia after every conceivable source of sepsis had been investigated and presumably eliminated and everything in the category of conventional therapy had been given a trial. However, I vaguely felt that existing methods of ridding the colon of toxic substances, correcting stasis, and re-establishing a normal flora were inadequate, and therefore decided to experiment a little with methods less commonly used, in an attempt to overcome this inadequacy. Colon irrigations as usually employed had, in the past, proved as useless as every other attempt to permanently influence sepsis in the bowel. Nevertheless, in principle, this approach appealed to me as being the most rational so far considered. The fact that it had heretofore failed might conceivably have been due to improper technique either of single irrigations or of a series, an erroneous conception of colon physiology leading to confusion as to what the irrigations were supposed to accomplish, and finally omission of important auxiliary and follow-up treatment and management.

I determined, therefore, to investigate the possibilities of this approach. After reviewing the various methods used in irrigating the colon, and after conversations with several physicians and technicians who had had some experience with them, and with inventors and manufacturers of colonic therapy apparatus, I began to understand the reasons for the current professional taboo and ridicule of this procedure. Whereas colon irrigations have always been used by physicians for the purpose of thoroughly cleansing the bowel, they rarely if ever had been employed as a therapeutic remedy for a large variety of diseases or as a cure-all for practically every disease that exists. I learned that many quacks and charlatans had for some time been exploiting this modality, thereby giving it a very unsavoury reputation. The natural reaction of the profession to such abuse was to have nothing to do with colonic therapy whatsoever. So pronounced was this 
reaction that but a very few physicians dared brave the criticism and ridicule that $\frac{2}{2}$ serious scientific interest in this subject invariably brought forth. A search of the $\stackrel{D}{2}$ literature failed to reveal anything of value on the subject. This field, therefore, $\stackrel{c}{-}$ from the scientific point of view has remained practically untouched.

It became very clear that any serious research into the value of colon irriga- $\frac{}{5}$ tions must be undertaken in the face of active antagonism. However, so thoroughly $\frac{\sigma}{\bar{\sigma}}$ was I convinced that the principle of free drainage was basically sound where $\frac{\vec{\sigma}}{\widehat{D}}$ chronic infection involving the bowel was concerned that I determined to find out why irrigations had failed in the past and what must be done to make them effective. $\stackrel{\text { s }}{\circ}$ It seemed very unreasonable to me that a method of such potential value should $\overrightarrow{0}$ be completely discarded for the sole reason that it was being exploited by irregulars. $\vec{\omega}$ To this end, I installed a simple unit, flexible enough in construction to serve the operator in the accomplishment of any desired technique.

This apparatus consisted essentially of several tanks elevated $2 \frac{1}{2}$ feet above $\stackrel{3}{.}$ the patient who reclined on a table on his left side. Rubber tubing from these $\overrightarrow{\vec{T}}$ jars, each equipped with a separate shut-off valve, led to a common inflow tube $\vec{N}$ equipped with a three-way valve which controlled the solutions entering and leaving $\sigma$ the bowel. The effluent discharges were directed to a slop jar or to the sewer. The rectal tube was of rubber with staggered openings near the tip. One jar contained the irrigation solution, the other solutions for instillation.

Patients selected for treatment were those still showing definite toxæmia after $\stackrel{\frac{D}{5}}{2}$ all known foci of infection had either been removed or thoroughly drained. Believing that sepsis in the bowel should be treated like any other open infectiong $\overrightarrow{0}$ I irrigated till the return flow showed but little fæcal material, then introduced soothing, mildly antiseptic solution at a temperature of 45 to 50 degrees $C$. The patient was then allowed to sit up and expel this. This procedure was followed daily for six days, then every other day until twelve treatments in all had been given. After this, treatments were given at lengthening intervals until all signs of toxæmia had completely disappeared. In the great majority of cases the above regime brought about the desired result, proving conclusively to me that I was justified in my conviction that this was the most rational approach to successful therapy of colon sepsis. However, this was only a beginning.

After twelve years of intensive study and practice in this field of low-grade toxæmias of bacterial origin I have learned to recognize many signs and symptoms which previously had been meaningless to me. Much of what I have learned has been recorded in my book on this subject and cannot be discussed here. It must 3 . be said, however, that a correct selection of cases, properly given irrigations, and $\delta$ subsequent management of these cases calls for prolonged study and experience, and must be considered a speciality in itself, not to be undertaken lightly even 9 by well qualified physicians. These patients cannot be turned over unreservedly to nurses or even trained technicians in the employ of the physician. The physician himself must follow each case daily since many predisposing and influencing causes $\tilde{\sigma}$ frequently appear unexpectedly and might be missed by a technician.

Failure by ignorant and unscrupulous quacks to produce permanent results is due largely to their ignorance of colon physiology and pathology and inability to 0 adapt technique to the case at hand. Unquestionably some patients are benefited o by these irregulars, but this is due to the working of the law of averages rather than to the intelligent recognition of indications for this method of therapy. Prospective patients for this type of treatment require careful study and special auxiliary treatment and subsequent management which those without medical 
knowledge are unable to give. The treatment of intestinal toxæmia by colonic therapy and auxiliary measures demands the personal attention of a thoroughly trained and experienced physician, and is doomed to failure in the hands of a quack in more than 75 per cent. of cases treated. On the other hand, the percentage of cures in the hands of a qualified physician with full co-operation of the patient will run well over 90 per cent.

The patient should be fully appraised of all the information the physician acquires concerning his case the background and mechanism of development of the toxæmia, the predisposing causes operative in causing it to become chronic, pitfalls he must avoid in the future, the diet he must follow, and habits he must form or break if he is to avoid relapse. It must be realized by both physician and patient that a series of colon irrigations, though greatly facilitating permanent cure, comprise only a small part of the therapeutic regime as a whole.

When the pathogenic organism causative of rheumatoid arthritis has established itself in the bowel, the treatment of this disease, regardless of what other therapy is being employed, must include the regime outlined for intestinal toxæmia, which, indeed, from the bacteriologic and therapeutic point of view it has become. Though exhibiting few or none of the symptoms of a systemic or intestinal toxæmia, the origin of infection, the mechanism of extension, the development of vicious circles and the tendency to chronicity are the same. The distinguishing characteristics, only, differ somewhat, and to this extent call for slight variations in routine management.

When a patient with rheumatoid arthritis comes to a physician for treatment, the history of the case should bring out the duration of the disease, previous treatment and operations for removal of foci, predisposing and initiating causes so far as they can be discovered, present status of activity of the disease, present habits and activity of the patient, number of joints involved, present diet, condition of the bowels, the presence or absence of pain and swelling, etc. A blood sedimentation test should be made at once, blood pressure taken and urine examined. If the disease is active as indicated by a rapid sedimentation rate, pain and swelling of the joints, loss of weight, exhaustion, nervous irritation, etc., all active treatment should be postponed with the exception of colon irrigations. These should be given daily for a week, using such solutions as Neo-Silvol, potassium permanganate, Zonite, pathogenicide, and Suldox as instillations. Except when coming to the physician's office the patient should remain in bed, or at least off his feet. After the first week irrigations should be given every other day for two weeks, then twice weekly, and finally once a week for a long time.

Acidophilus cultures should not be given as this does not influence streptococci, the organism usually responsible for the disease. The instillations should not be over 45 degrees $C$. as heat does not injure the streptococci and may actually cause undesirable reactions. Blood sedimentation tests should be made weekly to check the activity of the disease. This test is considered the most useful test in making an early differential diagnosis and in estimating activity of the disease (Hench, I939). Vaccines should not be used till activity has greatly subsided. Foci of infection should not be removed until a remission has been brought about and until vaccine has been used for a number of weeks. Before surgically removing foci, vaccine treatments should be temporarily discontinued, to be resumed later beginning with decreased dilutions. Complete rest is essential at first. Later, when activity of the disease has noticeably lessened, mild heat, passive exercise, and careful massage may be used tentatively. Massage should never be applied directly 
to the affected joints, but to the soft tissues above and below these joints only. Weekly irrigations should continue for months or a year or two if necessary, or until all indications of activity have completely ceased. Diet should be adequate, with low carbohydrate, high vitamin (particularly $B$ and $G$ ), and no roughage or irritating foods or laxatives.

Intestinal antispasmodics and bulk laxatives help to keep the colon free from bacteria and toxic substances and will prevent stasis. These aids should be selected to suit the requirements of the patient as no two patients respond alike. The patient should not be permitted to use enemas at home under the mistaken idea that they will accomplish the purpose of skillfully given irrigations. At best they empty the rectum and pelvic colon only, whereas a properly given irrigation cleanses and empties the entire colon from cæcum down.

Lest the reader form the impression that the author believes that colonic therapy is absolutely essential to permanent cure of rheumatoid arthritis when the colon is definitely involved, the author need only assure him that he is well aware of the many recognized cures in which no irrigations were used at all. However, in the author's experience, and in that of several well-known specialists with whose work he is acquainted, the use of colonic therapy is a measure of proved merit, bring ing about cures in cases of longstanding where all other measures had failed and in hastening the cure in cases where improvement had been very slow. Lieb (I93I), rhetorically inquires: "Who has not noticed the beneficial effects of colonic therapy on the progress of many cases of-arthritis?" Snyder (I939), remarks: "Colonic therapy is becoming an increasingly employed method in the management of arthritis, and rightly so, for there is no doubt that, in a great number of cases thio mode of treatment is highly successful." And Sexton (I934) asserts: "Strepto coccus and staphylococcus are the chief offending organisms in the production of -arthritis, - and influence that vast group of cases from whom we extirpate tonsils; teeth, appendices, gall-bladders, most of whom have improved with surprising speed when we treated their bowels."

Fishbaugh (I938) selected a group of I40 private patients with chronic arthritis in whom all factors except the colon had been excluded. The determination of the colon as the etiological factor to the chronic arthritis in this group of patients was based upon the following: I. History suggesting a relationship between the colon and the chronic arthritis. 2. Careful elimination of all other possible etiological factors. 3. The findings of colon abnormalities, such as active chronic constipation, delayed bowel movement type of constipation, chronic or subacute colitis, amœebiasis, or diverticulosis. 4. The relief of symptoms by therapy directed toward the correction of the colon dysfunction and disease.

Fishbaugh states that the treatment of chronic arthritis of the colon type is a 9 long procedure, that the patient must have great patience and continue the treatment for weeks, months or even years if necessary, and that relaxation in the colnn hygiene will result in a recurrence of the arthritis. Since the colon cannot be removed it must be kept well. He classified his results as quiescent, improved and not improved, and tabulated them as follows:-

Classification.

Quiescent

Improved

Unimproved
Number of CASEs.

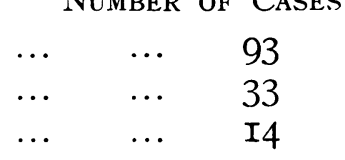

(Time in months)

Observation and Treatment.

... $\quad$... 36.0

I.9 
Among other measures colon irrigations were used extensively, the average number given each patient being 43 . One patient received 407 colon treatments. Traut states that arthritis requires a longer period for bacterial change than any other group of patients, and Goldfain in his discussion of Fishbaugh's paper remarks that it is quite common for symptoms to be more noticeable when good drainage of the colon is not present.

- It is significant that Wyatt (I933) whose patients come to him after an average duration of the disease of seven years finds colon irrigations of great value. He writes: "Colonic irrigations are exceedingly valuable hydrological measures and should occupy a much more important place in the treatment of the arthritides for the reasons set forth in connection with their etiology. Like analgesics, colonic irrigations have seemed to suffer either from neglect or abuse, with the result that it has been very difficult for the physician to 'steer a middle course' and give his patients the benefits to be derived from a form of therapy that is most effective when employed in carefully selected cases, with proper equipment and in accordance with sound principles. Experience has demonstrated that many (patients) who present manifestations of toxæmia and whose arthritis has resisted other treatment methods, respond rapidly to a therapeutic combination in which colonic irrigations play an important rôle." Burbank believes that properly given colon irrigations are the most satisfactory means we have of freeing the colon of toxic substances. Pemberton (I929) testifies that "With appropriate co-ordination of measurescolonic irrigation may achieve some of the most gratifying results to be obtained."

The following case from my own files would appear to indicate that the bowel was outstanding as a source of sepsis influencing this disease. J. C. A., male, married, age 52, civil engineer. This man came to my office October 4th, 1938, suffering from marked arthritis of the cervical and upper dorsal vertebræ and of both feet. The duration of the arthritis at this time was two years. He had been under the care of several different physicians during this period with no apparent benefit. On the contrary the disease had progressed steadily and he was rapidly becoming crippled. Several weeks before coming to me he had been through an extensive diagnostic study by a local internist who finally admitted he could locate no focus of infection or find anything that could be interpreted as an etiological factor. At this time his blood pressure was, systolic 112, diastolic 70. Prostate was moderately enlarged but yielded no abnormal secretion. His teeth were in good repair. His tonsils were reported normal. The urine was normal except for an increased amount of indican. The Wasserman reaction was negative. Blood chemistry findings were normal. Gastric analysis was normal as to hcl, lactic acid, bile, blood and bacteria. The blood count showed 4,160,000 red cells, 11,000 leucocytes, hæmoglobin 70 per cent. (11 gms. per 100 c.c.), neutrophiles 78 per cent. (72 per cent. segmented), lymphocytes 22 per cent. X-ray of spine, lateral aspect showed considerable bone atrophy of all ventebral bodies of cervical and upper two thoracic vertebræ with marked bridging across the intervertebral spaces, especially in the region of the sixth and seventh cervical and first and second thoracic, which appeared practically fused. There was marked spur formation and ankylosing arthritis of the vertebræ in question. The bones of the feet showed no pathology.

Further points brought out by my examination were: Indican in urine very heavy, blood sedimentation rate $65 \mathrm{~mm}$. in one hour, both feet swollen and very tender. Walking caused him considerable pain. Muscles of shoulder girdle very spastic and sensitive. He could not turn his head at all without severe pain. Pressure over spinous processes of cervical and upper dorsal vertebræ caused much pain. Swelling was marked. Examination of the bared abdomen showed marked distension, considerable gas in hepatic and 
splenic flexures, a loaded transverse colon, markedly distended cæcum and a rigid spastic pelvic colon. He had been troubled with periodic attacks of constipation accompanied with severe headaches. A mild laxative always relieved this condition. $\mathrm{He}$ took no enemas or laxatives except during these attacks which came every three or four weeks.

Treatment consisted of daily colon irrigations for one week, then every other day for two weeks, then twice weekly for many weeks. He is now, June I939, receiving one irrigation weekly. Blood sedimentation rate Oct. 4th, I938, was $65 \mathrm{~mm}$. in an hour; Oct. IIth, $55 \mathrm{~mm}$.; Oct. I8th, $37 \mathrm{~mm}$.; Oct. 25th, $30 \mathrm{~mm}$. It remained at $30 \mathrm{~mm}$. for some weeks. On Nov. 3rd, an aqueous solution of sulphur dioxide was given, to be taken in increasing amounts four times daily till maximum tolerance was reached, then diminished in dosage to a point at which no disagreeable symptoms (increased tissue tenderness) followed its administration. This solution (Suldox) was also used in some of the colon instillations. On Nov. 26th, the sedimentation rate had fallen to $15 \mathrm{~mm}$. in an hour, at which point it has become stationary. There has been no other therapy except an antispasmodic, a bulk laxative consisting of the mucilagenous component of psyllium seed and finely divided kapok fibre (proprietary name, Fibrosyl), and mild short wave diathermy to the feet, begun late in October and discontinued in December. The Alvarez smooth diet was used but there was no change made in his daily routine of active work. In January, I939, he took a vacation of one month in Florida.

From the beginning of treatment in October, the patient has shown steady objective and subjective improvement until at the present time all pain has disappeared, all swelling gone, he walks without pain or difficulty and the only persisting symptom is a stiffness of the muscles of his shoulder girdle which I believe will yield to mild short wave diathermy and massage. He has had but one headache in more than eight months.

The patient's tonsils were removed in December without causing a reaction. There had been nothing about them to cause suspicion and no cultures were made. It was just a precautionary measure. In April, weekly injections of a stock vaccine were begun (Lederle's rheumatoid arthritis vaccine), intravenously. To date nine of these have been given with no reaction at any time. It is too early yet to claim a cure, yet improvement has been steady and marked and still continues. This case has been selected deliberately to indicate the factor of colon sepsis and its management in rheumatoid arthritis.

REFERENCES.

ALBEE, FRED. H. (1928), J.A.M.A., 91, 1364.

BURBANK, REGINALD (1935), Discussion of Dr. Gutman's Paper, “The Arthritides and Colon Therapy," Arch. Phys. Therapy, 16, 178

COHEN, ABRAHÁM (1937), Internat. Clin., 3, 287.

CROWE, H. W. (1937), Med. Press, 194, 426.

FISHBAUGH, E. C. (1938), "Colon Disease and its Therapy in Relation to Chronic Arthritis." Read before the Convention of the American Congress of Physical Therapy, Chicago (Sept. 13th).

GOLDFAIN, EPHRAIM (1939), "Importance of Gastrointestinal Therapy in the Control or Cure of Arthritis," Arch. Phys. Therapy, 20, 357.

HARKAVY, JOSEPH and HEBALD, SILIAS (1932), Arch. Int. Med., 49, 698.

HENCH, P. 'S., et al., "The Problem of Rheumatism and Arthritis." Review of American and English Literature for 1937, Annals of Int. Med., 12, 1005.

HURST, ARTHUR F. (1919), " Constipation and Allied Intestinal Disorders," Oxford Medical Publications (London).

LAUTMAN, MAURICE F. (1938), Arch. Phys. Therapy, 19, 426.

LIEB, CLARENCE W. (1931), New York State J. Med., 31, 805.

LIEB, CLARENCE W. and CHAPMAN, GEO. H. (1929), "Studies in Intestinal Intoxication," New York State J. Med.,

PEMBERTON, RALPH (1929), "Arthritis and Rheumatoid Conditions," Lea and Febiger, Philadelphia.

PEMBERTON, RALPH (1932), Surg., Gynæc. and Obst., 54, 333.

SEXTON, ROY LYMAN (1934), Arch. Phys. Therapy, 15, 489.

SNYDER, R. G. and TRAEGER, C. H. (1930), J. Lab. Clin. Med., 15, 1214.

TRAUT, EUGENE F. (1934), Arch. Phys. Therapy, 15, 479.

WILTSIE, JAMES W. (1938), "Chronic Intestinal Toxæmia and Its Treatment," William Wood and Co. Baltimore, Maryland.

WYATT, BERNARD L. (1933), “Chronic Arthritis and Fibrositis," William Wood and Co. Baltimore, Maryland.

ZINSSER, H. and GRINNEL, F. A. (1925), J. Immunol, 10, 725. 